\title{
A goal-based angular adaptivity method for thermal radiation modelling in non grey media
}

\author{
Laurent Soucasse $^{\mathrm{a}, *}$, Steven Dargaville ${ }^{\mathrm{a}}$, Andrew G. Buchan ${ }^{\mathrm{a}}$, Christopher \\ C. Pain ${ }^{\mathrm{a}}$ \\ ${ }^{a}$ Applied Modelling and Computation Group, Department of Earth Science and \\ Engineering, Imperial College London, SW7 2AZ, United Kingdom
}

\begin{abstract}
This paper investigates for the first time a goal-based angular adaptivity method for thermal radiation transport, suitable for non grey media when the radiation field is coupled with an unsteady flow field through an energy balance. Anisotropic angular adaptivity is achieved by using a Haar wavelet finite element expansion that forms a hierarchical angular basis with compact support and does not require any angular interpolation in space. The novelty of this work lies in (1) the definition of a target functional to compute the goal-based error measure equal to the radiative source term of the energy balance, which is the quantity of interest in the context of coupled flowradiation calculations; (2) the use of different optimal angular resolutions for each absorption coefficient class, built from a global model of the radiative properties of the medium. The accuracy and efficiency of the goal-based angular adaptivity method is assessed in a coupled flow-radiation problem relevant for air pollution modelling in street canyons. Compared to a uniform
\end{abstract}

\footnotetext{
${ }^{*}$ Corresponding author.

Email address: 1.soucasse@imperial.ac.uk (Laurent Soucasse)
} 
Haar wavelet expansion, the adapted resolution uses 5 times fewer angular basis functions and is 6.5 times quicker, given the same accuracy in the radiative source term.

Keywords: goal-based adaptivity, angular adaptivity, coupled flow-radiation, non grey media

\section{Introduction}

Numerical approximations to the Radiative Transfer Equation (RTE) remain a computational challenge and an intense research area. The difficulty comes from the dependence of the radiative intensity in space and angle, in time when the radiation field is coupled with an unsteady flow, and in wavenumber as the absorption spectrum of common radiating gases like water vapour or carbon dioxide are made of millions of lines. The emergence of parallel computing has made the use of detailed reference methods for solving the RTE, such as the Monte Carlo method or the ray-tracing method, more and more practical. The coupling of these reference methods with a Direct Numerical Simulation (DNS) of a turbulent flow has been for instance recently achieved in Ref. [1]. However, a strong interest persists in reducing the dimension of the discretised RTE for rapid modelling and engineering

purposes as well as for simulating large scale systems out of the scope of reference methods.

A substantial effort has been devoted in the literature to reduce the dimension of the wavenumber space and has lead to the development of correlated- $k$ models, Statistical Narrow Band models or global models. A review of these different approaches is given by Taine and Soufiani [2]. Global 
models, which consist in reordering the absorption spectrum according to the value of the absorption coefficient over the whole spectral domain, are the most computationally efficient and are favoured for 3D applications, although they suffer from inaccuracies in heterogeneous media.

In the thermal radiation community, the angular and spatial discretisation are most often achieved by combining the Discrete Ordinates Method (DOM or $S_{N}$ expansion) in angle with the Finite Volume Method (FVM) in space $[3,4]$, although the finite element method also provides a general framework for both the angle and space dimensions [5]. A promising way to reduce the dimension of these discretised space-angle systems is the use of adaptive discretisation methods that only refine the regions of interest. These adaptive algorithms are driven by error estimators and include regular adaptive methods where the solution error is reduced uniformly over the whole phase-space domain and goal-based adaptive methods where the solution error is reduced with respect to a target functional.

Several spatial adaptivity schemes for radiation transport havehas been developed during the past decade. An Adaptive Mesh Refinement (AMR) technique, based on a hierarchy of structured spatial meshes, was used by Ogando and Velarde [6], combined with a $S_{N}$ expansion in angle. Ragusa [7] proposed a regular error measure, using the Hessian of the discretised spatial flux, while Lathouwers [8] and Goffin et al. [9] experimented with goalbased error measures with various target functionals. More recently, Yang and Yuan [10] developed a $h$-refinement technique for simple corner balance scheme for application to radiation transport coupled with Lagrangian hydrodynamics. Although all these space adaptivity techniques are successful 
in reducing the number of spatial degrees of freedom, their efficient implementation in parallel remains challenging.

A first attempt at adapting the angular resolution was proposed by Ackroyd and Wilson [11] who used a variable order spherical harmonic expansion $\left(P_{N}\right)$ across space using an a priori knowledge of the spatial variation of the physical properties of the medium. Using a $P_{N}$ expansion is advantageous for angular adaptivity because it forms a hierarchical basis and it does not need any angular interpolation in space. Goffin et al. took advantage of this property to develop goal-based angular adaptivity algorithms using a $P_{N}$ expansion [12]. Alternative hierarchical basis for angular discretisation can be formed using wavelet theory and multi-resolution analysis [13]. Compared to spherical harmonics, wavelets have the advantage of adapting anisotropically due to their compact support. Watson [14] and Goffin et al. [15] both made use of wavelet-based angular adaptivity methods with Haar wavelets and octahedral linear wavelets respectively. Other adaptive schemes have also been developed for non-hierarchical basis, such as the $S_{N}$ expansion [16]. Kópházy and Lathouwers [17] proposed a generalised framework for local angular refinement with arbitrary angular basis functions and discontinuous Galerkin discretisation in space and angle, but the method involves complex algebra to compute the numerical fluxes. Moreover, alternative techniques exist in order to reduce the angular dimension, such as reduced order models [18, 19].

Most of these angular adaptivity methods have been applied for problems in nuclear engineering and have not been applied in problems with thermal radiation. In many engineering or environmental applications, the thermal radiation field is coupled with a flow field through an energy balance. In 
that case the key quantity to predict is the radiative source term, namely the balance between the radiation absorbed and emitted in the medium. In this paper, we apply angular adaptivity for problems in thermal radiation and derive a goal-based adaptivity method that optimises the angular resolution to accurately compute the radiative source term. In order to perform anisotropic angular adaptivity, we make use of a Haar wavelet angular expansion, which is a hierarchical version of a $S_{N}$ expansion and is compactly supported. The angular dependence of the radiation field strongly depends on the optical thickness of the medium, and hence we apply different angular resolutions for different absorption coefficient classes built from a global model of the radiative properties of the medium.

The layout of the article is as follows. The numerical methods and models we use to discretise the RTE are described in Sec. 2 and the goal-based adaptivity algorithm is presented in Sec. 3. The accuracy and efficiency of our goal-based angular adaptivity method is assessed in Sec. 4 in a coupled flow-radiation problem relevant for air pollution modelling in street canyons.

\section{Radiative tranfer equation and numerical methods}

We consider in this work a non scattering medium of optical index equal to 1 at local thermal equilibrium, hence we can write the RTE as

$$
\boldsymbol{\Omega} \cdot \boldsymbol{\nabla} I(\nu, \boldsymbol{r}, \boldsymbol{\Omega})=\kappa(\nu)\left(I_{b}(\nu, T(\boldsymbol{r}))-I(\nu, \boldsymbol{r}, \boldsymbol{\Omega})\right)
$$

where $I(\nu, \boldsymbol{r}, \boldsymbol{\Omega})$ is the radiative intensity at point $\boldsymbol{r}$, direction $\boldsymbol{\Omega}$ and wavenumber $\nu$ and $I_{b}(\nu, T(\boldsymbol{r}))$ is the blackbody intensity at local temperature $T(\boldsymbol{r})$. The absorption coefficient $\kappa(\nu)$ is assumed to be homogeneous for the prac- 
tical case addressed in Section 4, although this is not a restriction of the numerical methods and the adaptivity algorithm presented in this paper. The associated boundary condition for a diffuse opaque wall of grey emissivity $\varepsilon$ is

$$
I\left(\nu, \boldsymbol{r}_{w}\right)=\varepsilon\left(\boldsymbol{r}_{w}\right) I_{b}\left(\nu, T\left(\boldsymbol{r}_{w}\right)\right)+-\frac{1-\varepsilon\left(\boldsymbol{r}_{w}\right)}{\pi} \int_{\boldsymbol{\Omega}^{\prime} \cdot \boldsymbol{n}<0} I_{\nu}\left(\boldsymbol{r}_{w}, \boldsymbol{\Omega}^{\prime}\right)\left|\boldsymbol{\Omega}^{\prime} \cdot \boldsymbol{n}\right| d \boldsymbol{\Omega}^{\prime},
$$

for points $\boldsymbol{r}_{w}$ and directions $\boldsymbol{\Omega}$, such that $\boldsymbol{\Omega} \cdot \boldsymbol{n}>0, \boldsymbol{n}$ being the wall normal directed towards the inside of the domain.

The radiative source term is equal to the opposite of the divergence of the radiative flux $\boldsymbol{q}^{\text {rad }}$

$$
-\boldsymbol{\nabla} \cdot \boldsymbol{q}^{\text {rad }}(\boldsymbol{r})=\int_{\boldsymbol{\Omega}} \int_{\nu} \kappa(\nu)\left(I(\nu, \boldsymbol{r}, \boldsymbol{\Omega})-I_{b}(\nu, T(\boldsymbol{r}))\right) d \nu d \boldsymbol{\Omega} .
$$

The radiative source term goes into the energy balance of the material system: it represents the difference between the absorption and emission of radiative energy by the medium. It is this key quantity that we will try to optimise with the adaptive algorithm

\subsection{Radiative property modelling}

In order to model the spectral dependence of the absorption coefficient, we make use of a global model based on an absorption distribution function [20] defined by

$$
\mathcal{F}(k)=\frac{\pi}{\sigma T_{\text {ref }}^{4}} \int_{\nu, \kappa_{\nu}\left(T_{\text {ref }}\right) \leq k} I_{b}\left(\nu, T_{\text {ref }}\right) d \nu .
$$

This function is discretised in intervals $\left[k_{i}^{-} ; k_{i}^{+}\right]$of averaged value $k_{i}$. The weights of this distribution associated with each interval $i$ are defined as $a_{i}=\mathcal{F}\left(k_{i}^{+}\right)-\mathcal{F}\left(k_{i}^{-}\right)$. Equations (1) and (2) then become

$$
\boldsymbol{\Omega} \cdot \boldsymbol{\nabla} I_{i}(\boldsymbol{r}, \boldsymbol{\Omega})=k_{i}\left(\frac{a_{i} \sigma T^{4}(\boldsymbol{r})}{\pi}-I_{i}(\boldsymbol{r}, \boldsymbol{\Omega})\right),
$$




$$
I_{i}\left(\boldsymbol{r}_{w}\right)=\varepsilon\left(\boldsymbol{r}_{w}\right) \frac{a_{i} \sigma T^{4}\left(\boldsymbol{r}_{w}\right)}{\pi}+-\frac{1-\varepsilon\left(\boldsymbol{r}_{w}\right)}{\pi} \int_{\boldsymbol{\Omega}^{\prime} \cdot \boldsymbol{n}<0} I_{i}\left(\boldsymbol{r}_{w}, \boldsymbol{\Omega}^{\prime}\right)\left|\boldsymbol{\Omega}^{\prime} \cdot \boldsymbol{n}\right| d \boldsymbol{\Omega}^{\prime}
$$

where $\sigma$ is the Stefan-Boltzmann constant. The total intensity integrated over the wavenumber is simply retrieved by summing the contribution of each $k$-class: $I=\int I(\nu) d \nu=\sum_{i} I_{i}$.

This global model is advantageous because the integration over the wavenumber is replaced by an integration over the absoption coefficient $k$, for which a coarse discretisation is sufficient. Another benefit is that we will be able to associate to each $k$-class a different angular resolution, as the angular dependence of the radiative intensity is known to be affected by the optical thickness of the medium. As a homogeneous medium is considered, the derivation of Eqs. (5)-(6) is exact and the model error is associated with the numerical discretisation in $k$. However, additional assumptions are required to extend the model to heterogeneous media (see for instance Refs. [21-23]). The model parameters for the medium considered in Sec. 4 are given in Appendix A.

\subsection{Space-angle finite element discretisation}

A Sub-Grid Scale (SGS) model (or variational multiscale method, see Ref. [24]) is used to discretised the RTE in space. The full solution $I_{i}(\boldsymbol{r}, \boldsymbol{\Omega})$ is decomposed into a coarse component $\bar{I}_{i}(\boldsymbol{r}, \boldsymbol{\Omega})$ and a sub-grid component $\tilde{I}_{i}(\boldsymbol{r}, \boldsymbol{\Omega})$, both approximated by a finite element formulation. The coarse component lies in a continuous finite element space, spanned by $\eta_{N}$ basis functions, and the sub-grid component lies in a discontinuous space, spanned 
by $\eta_{Q}$ basis functions, as follow

$$
I_{i}(\boldsymbol{r}, \boldsymbol{\Omega}) \simeq \sum_{j=1}^{\eta_{N}} N_{j}(\boldsymbol{r}) \bar{I}_{i j}(\boldsymbol{\Omega})+\sum_{j=1}^{\eta_{Q}} Q_{j}(\boldsymbol{r}) \tilde{I}_{i j}(\boldsymbol{\Omega}) .
$$

The continuous basis functions $N_{j}(\boldsymbol{r})$ and the discontinuous basis functions $Q_{j}(\boldsymbol{r})$ are associated with the same mesh and are both piecewise linear across the elements in this work.

An arbitrary discretisation of the angular dimension is considered in which the angular space is represented by the span of $\mathcal{M}$ angular basis functions $G_{q}(\Omega)$ such that

$$
\bar{I}_{i j}(\boldsymbol{\Omega}) \simeq \sum_{q=1}^{\mathcal{M}} G_{q}(\boldsymbol{\Omega}) \bar{I}_{i j q}, \quad \widetilde{I}_{i j}(\boldsymbol{\Omega}) \simeq \sum_{q=1}^{\mathcal{M}} G_{q}(\boldsymbol{\Omega}) \tilde{I}_{i j q}
$$

The same set of angular basis functions is used for both the coarse and subgrid spatial components and we will assume for now that this set is uniform in space and for each $k$-class $i$.

Introducing the decompositions (7)-(8) into the RTE and applying a Galerkin projection in space and angle leads to a space-angle block linear system of the form

$$
\left(\begin{array}{ll}
\mathrm{A} & \mathrm{B} \\
\mathrm{C} & \mathrm{D}
\end{array}\right)\left(\begin{array}{l}
\bar{I} \\
\tilde{I}
\end{array}\right)=\left(\begin{array}{c}
\overline{\mathrm{S}} \\
\tilde{\mathrm{S}}
\end{array}\right)
$$

that has to be solved for each $k$-class $i$. The definition of the submatrices A, $\mathrm{B}, \mathrm{C}, \mathrm{D}$ and the source terms $\overline{\mathrm{S}}, \tilde{\mathrm{S}}$ can be found in [25]. The sub-grid solution can be formally written as

$$
\tilde{I}=-\mathrm{D}^{-1} \mathrm{C} \bar{I}+\mathrm{D}^{-1} \tilde{\mathrm{S}}
$$

Using Eq. (10), we can form a single linear system for the coarse component

$$
\left(\mathrm{A}-\mathrm{BD}^{-1} \mathrm{C}\right) \bar{I}=\overline{\mathrm{S}}-\mathrm{BD}^{-1} \tilde{\mathrm{S}}
$$


involving the Shur complement of the block matrix D.

The role of the sub-grid component is to suppress any instability and non-physical oscillations that may arise from a continuous finite element discretisation of the RTE. Therefore, we can approximate the computation of matrix D associated with the discontinuous sub-grid component, which is the largest matrix involved. To make D more sparse and make its inversion efficient, the solution of the sub-grid scale is restricted to be strictly local to each element and the coupling between angular moments in an element is neglected. However, no assumptions are made for the computation of matrices A, B and C. A thorough review of the implementation of the SGS model, its accuracy and its efficiency, is provided in Refs. [25-27]. We should note that this sparsification of $\mathrm{D}$ gives different amounts of stabilisation between different angular discretisations. This is particularly relevant in Sec. 2.4, as we make comparisons between $S_{N}$ and Haar wavelets, and though these quadratures are equivalent, our discretisation given the sparse form of D are not (though the differences are very small; they are caused purely by the differences in neglected coupling between angular moments in the Haar hierarchy, see Section 2.3).

Boundary conditions are implemented using a Riemann decomposition method [28] to separate the angular dependence of the flux through a surface into incoming and outgoing information. The advantage of such a method is to work with any kind of angular discretisation (spherical harmonics, wavelets, etc.). For Haar wavelet angular discretisation, the Riemann decomposition is simple as we can rely on a mapping into an $S_{N}$ space (see Sec. 2.3). 


\subsection{Haar wavelet angular discretisation}

The angular adaptivity algorithm relies on the use of a hierarchical functional basis which means that the set of basis functions at an order $n$ is a sub-set of basis functions at an order $n+1$. This allows us to use different sets of angular basis functions of different orders across space without the need to perform angular interpolation.

The Haar wavelet expansion is a hierarchical version of the discrete ordinates $\left(S_{N}\right)$ expansion. The Haar wavelets are piecewise constant functions built from a multiresolution analysis (MRA) and associated with a partition scheme of the angular space [27, 29]. An exact linear mapping relates the Haar wavelet and the discrete ordinates expansions built from the same angular partition. Thus, a transformation matrix can be applied to angular matrices in order to map from one discretisation to the other. This simplifies the implementation of Haar wavelets, allowing them to be built on existing $S_{N}$-type frameworks, provided that those are derived from a hierarchical partition of the angular domain. A 1D representation of the Haar wavelet basis functions over an arbitrary interval is given in Fig. 1 with the corresponding $S_{N}$ basis functions and the transformation matrix. The angular matrices are less sparse with a Haar discretisation than with a $S_{N}$ discretisation, making them more expensive to apply, but this is balanced by the hierarchical nature of the Haar wavelets with which angular adaptivity is simple.

In this paper, the Haar wavelet space will be noted $W_{m, n}$ and will be associated to the following 2D partition of the angular space: in each octant, the values of the cosine of the polar angle $\mu$ are divided into $2^{m-1}$ equallyspaced intervals and the values of the azimuthal angle $\varphi$ are divided into 


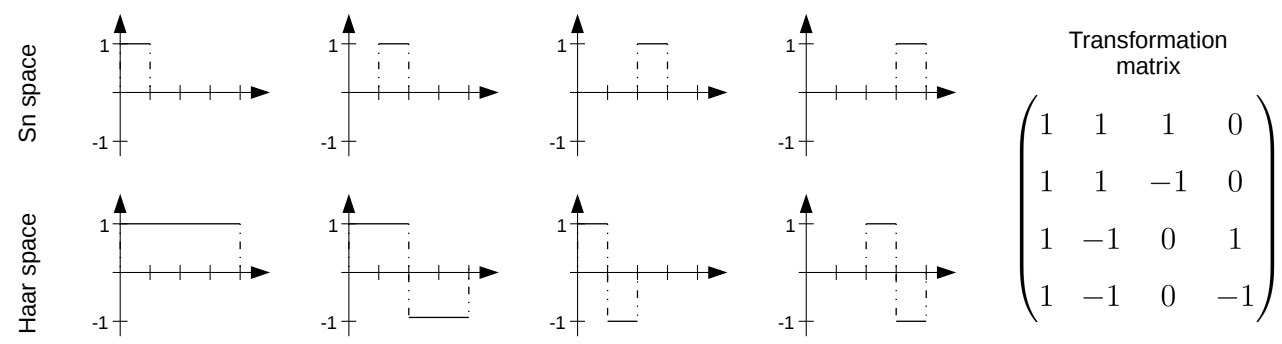

Figure 1: Left: representation of the Haar wavelet and $S_{N}$ basis functions over a 1D arbitrary interval at the order 3 (partition of the interval in 4). Right: the transformation matrix that relates the two spaces.

$2^{n-1}$ equally-spaced intervals, leading to a total number of basis function of $8 \times 2^{m-1} \times 2^{n-1}$ in three dimensions of space. The discrete ordinates space associated with the same angular partition will be noted $S_{m, n}$. The 2D Haar wavelets are obtained from a tensor product of the 1D Haar wavelets in the polar and azimuthal directions.

For a given maximum expansion Haar order, the adaptivity algorithm detailed in Sec. 3 only retains the wavelets of significant importance in relation to a target error. Therefore, the set of angular basis functions is allowed to vary in space and for each $k$-class, Eq. (8) becomes

$$
\bar{I}_{i j}(\Omega) \simeq \sum_{q \in \overline{\mathcal{M}}_{i j}} G_{q}(\Omega) \bar{I}_{i j q}, \quad \tilde{I}_{i j}(\Omega) \simeq \sum_{q \in \tilde{\mathcal{M}}_{i j}} G_{q}(\Omega) \tilde{I}_{i j q}
$$

where $\overline{\mathcal{M}}_{i j}$ and $\tilde{\mathcal{M}}_{i j}$ are respectively the coarse and sub-grid set associated to a spatial point $j$ and a $k$-class $i$. At a given point of space, we constrain the continuous and discontinuous components to be approximated with the same wavelet set, what we can formally write $\overline{\mathcal{M}}_{i j}=\tilde{\mathcal{M}}_{\mathcal{C}(j) i}, j \in\left\{1, \cdots, \eta_{Q}\right\}$, $\mathcal{C}$ being a surjective mapping from the continuous nodes to the discontinuous 
nodes. Although the Haar wavelet angular discretisation is combined in this work with a spatial SGS model, it can be used with any type of spatial discretisation, as well as the angular adaptivity algorithm.

\subsection{Implementation and validation}

The space-angle linear system is solved using the generalised minimal residual method (GMRES) with a spatial multigrid preconditioner [30]. Both GMRES and multigrid methods are implemented in a matrix-free fashion as the whole space-angle system is often too large to store in memory.

In order to validate the implementation of the space-angle discretisation methods with a uniform Haar wavelet expansion we have reproduced the 3D benchmark test case proposed by Soucasse et al. [31]. A cubic cavity of size $L$ is considered, enclosed by opaque walls of emissivity $\varepsilon=0.5$ and filled with a grey participating medium of absorption coefficient $\kappa$ such that $\kappa L=1$. A Gaussian temperature field $T(x, y, z)=\exp \left(-\left(x-x_{c}\right)^{2}-(y-\right.$ $\left.\left.y_{c}\right)^{2}-\left(z-z_{c}\right)^{2}\right) \Delta T+T_{0}$ is prescribed in the medium with $\left(x_{c}, y_{c}, z_{c}\right)=$ $(0.25 L, 0.25 L, 0.25 L), \Delta T=10 \mathrm{~K}$ andet $T_{0}=300 \mathrm{~K}$. The walls are isothermal at the temperature $T_{0}$. This problem possesses a reflection symmetry along the line $x=y=z$.

The reference results of Ref. [31] were obtained with the Monte Carlo method and calculations were performed on a uniform grid of $42^{3}$ cells using $6 \times 10^{9}$ stochastically generated optical paths. Our calculations have been

performed on an unstructured mesh made of 46,000 nodes and both with a uniform Haar wavelet angular discretisation of order $4\left(W_{4,4}\right.$, namely 512 angular basis functions) and a discrete ordinates discretisation using the same angular partition $\left(S_{4,4}\right)$. 

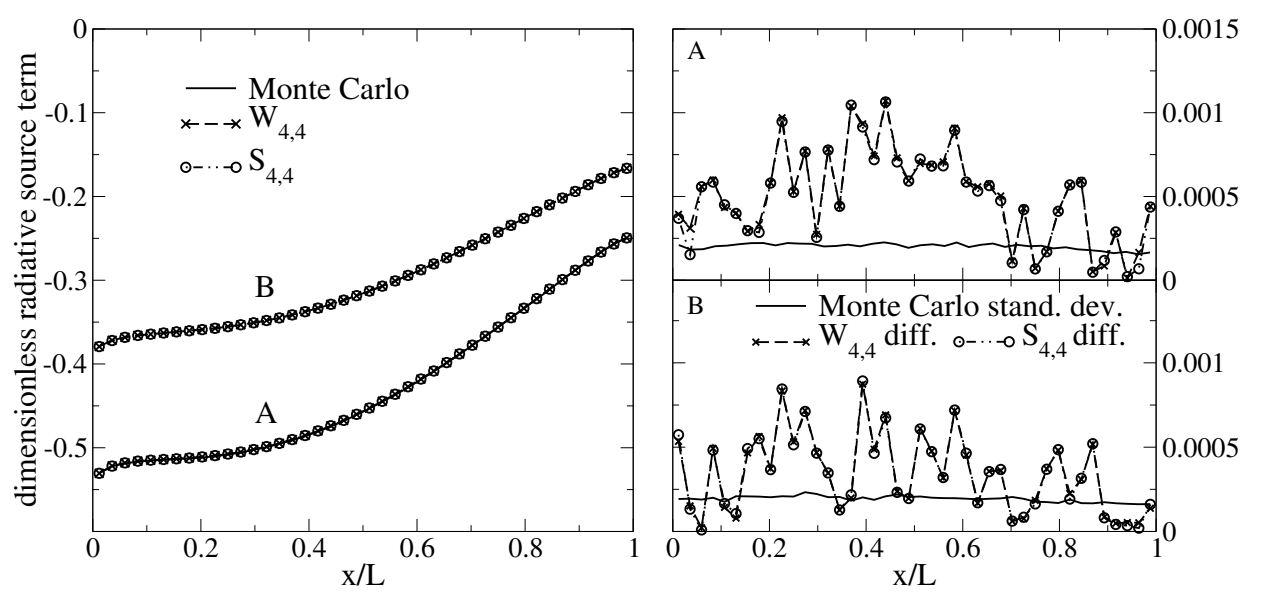

Figure 2: Benchmark test case of Ref. [31]. Left: radiative source term (Eq. (3)) made dimensionless by $4 \kappa \sigma\left(\left(T_{0}+\Delta T\right)^{4}-T_{0}^{4}\right)$ along the lines $y / L=0.25, z / L=0.25$ (A), and $y / L=0.25, z / L=0.75$ (B). Right: Monte Carlo standard deviation and absolute difference obtained with $W_{4,4}$ and $S_{4,4}$ compared to the Monte Carlo calculations along the same lines.

Figure 2 plots the radiative source term along two lines and the associated differences obtained with the $W_{4,4}$ and $S_{4,4}$ expansions compared to the Monte Carlo calculations. Both the $W_{4,4}$ and $S_{4,4}$ calculations agree with the Monte Carlo results: the differences do not exceed $0.25 \%$ while the standard deviation of the Monte Carlo calculations are of the order of $0.1 \%$. It can also be noticed that the two difference curves (using either $W_{4,4}$ or $\left.S_{4,4}\right)$ overlap. This is an expected results as the two expansions are related by a linear mapping. The minor differences that can be seen between our two angular discretisations are related to the stabilization scheme for which different approximations are applied, as mentioned in Sec. 2.2.

We have now validated the implementation of uniform Haar wavelet angular discretisation. In Sec. 4, the accuracy and efficiency of the angular 
adaptivity algorithm will be assessed by comparing adapted and uniform Haar wavelet expansions.

\section{Goal-based angular adaptivity}

The angular adaptivity algorithm used in this paper is driven by a goalbased error measure. The goal has the form of an arbitrary functional $F$ which is a target of physical interest that one wants to compute accurately. In the framework of coupled flow/radiation calculations, we are interested in controlling the error in the radiative source term defined in Eq. (3) which goes into the energy balance of the material system. We propose here to use the radiative source term integrated in space as a goal and thus the following functional will be considered

$$
F(I(\nu, \boldsymbol{r}, \boldsymbol{\Omega}))=\int_{\boldsymbol{r}} \int_{\boldsymbol{\Omega}} \int_{\nu} \kappa(\nu)\left(I(\nu, \boldsymbol{r}, \boldsymbol{\Omega})-I_{b}(\nu, T(\boldsymbol{r}))\right) d \nu d \boldsymbol{\Omega} d \boldsymbol{r} .
$$

\subsection{Functional error measure}

The goal-based error measure requires the solution of the RTE defined by Eq. (1) (further referred to as the forward problem) as well as the solve of an adjoint radiative transfer problem defined by

$$
-\boldsymbol{\Omega} \cdot \boldsymbol{\nabla} I^{*}(\nu, \boldsymbol{r}, \boldsymbol{\Omega})=\mathcal{S}^{*}-\kappa(\nu) I^{*}(\nu, \boldsymbol{r}, \boldsymbol{\Omega}),
$$

where $I^{*}(\nu, \boldsymbol{r}, \boldsymbol{\Omega})$ is the solution of the adjoint problem and $\mathcal{S}^{*}$ is the adjoint source term involving the targeted goal and given by

$$
\mathcal{S}^{*}=\frac{\partial f(I(\nu, \boldsymbol{r}, \boldsymbol{\Omega}))}{\partial I(\nu, \boldsymbol{r}, \boldsymbol{\Omega})}=\kappa(\nu),
$$

with $f$ being the integrand of the functional $F$ as defined in Eq. (13). 
Following Goffin et al. [12, 15], the functional error measure is expressed as the inner product in the phase space between the residual of the forward computation $\mathcal{R}(I)$ and the error in the adjoint computation

$$
F\left(I_{\text {exact }}\right)-F(I) \simeq-\int_{\boldsymbol{r}} \int_{\boldsymbol{\Omega}} \int_{\nu} \mathcal{R}(I)\left(I_{\text {exact }}^{*}-I^{*}\right) d \nu d \boldsymbol{\Omega} d \boldsymbol{r},
$$

where $I_{\text {exact }}^{*}$ denote the exact solution to the adjoint problem (Eq. (14)). The estimate provided by Eq. (16) is called the adjoint error measure. An alternative functional error measure is expressed as the inner product in the phase space between the residual of the adjoint computation $\mathcal{R}^{*}\left(I^{*}\right)$ and the error in the forward computation

$$
F\left(I_{\text {exact }}\right)-F(I) \simeq-\int_{\boldsymbol{r}} \int_{\boldsymbol{\Omega}} \int_{\nu} \mathcal{R}^{*}\left(I^{*}\right)\left(I_{\text {exact }}-I\right) d \nu d \boldsymbol{\Omega} d \boldsymbol{r}
$$

where $I_{\text {exact }}$ denote the exact solution to the forward problem (Eq. (1)). The estimate provided by Eq. (17) is called the forward error measure.

\subsection{Discrete error estimates}

For a practical use of the integral error measures given by Eqs. (16)-(17), a discrete form of the solution error and the residual has to be derived in relationship with the angular discretisation we want to adapt. We make use here of the heuristic expressions given by Goffin et al. [15] that take advantage of the hierarchical nature of the angular basis functions. The discrete solution error $\epsilon$ and residual $R$ are first computed for the full solution (union of the sub-grid and coarse components) in the discontinuous spatial space for both the adjoint and forward problems. These quantities are then mapped to the continuous spatial space by taking the maximum value at each spatial node. 
The adjoint and forward goal-based error measures are formally written as

$$
\begin{aligned}
\widehat{e}_{i j q}^{*} & =\frac{\left|\widehat{\epsilon}_{i j q}^{*} \widehat{R}_{i j q}\right|}{\Delta \widehat{F}}, \\
\widehat{e}_{i j q} & =\frac{\left|\widehat{\epsilon}_{i j q} \widehat{R}_{i j q}^{*}\right|}{\Delta \widehat{F}},
\end{aligned}
$$

where $\widehat{\epsilon}$ and $\widehat{\epsilon}^{*}$ are the forward and adjoint continuous solution errors, $\widehat{R}$ and $\widehat{R}^{*}$ are the forward and adjoint continuous residuals and $\Delta \widehat{F}$ is a normalising factor related to a user-defined tolerance for the functional goal. The indices $i, j$ and $q$ refer to the wavenumber, space and angular degrees of freedom and vary for $i=\left\{1, \cdots, N_{k}\right\}, j=\left\{1, \cdots, \eta_{N}\right\}$ and $q \in \overline{\mathcal{M}}_{i j}$. Adjoint $\widehat{e}_{i j q}^{*}$ and

forward $\widehat{e}_{i j q}$ error estimators are then combined to get a unique goal-based error measure $e_{i, j, q}^{\mathrm{GB}}$ such that

$$
e_{i, j, q}^{\mathrm{GB}}=\max \left\{\widehat{e}_{i j q}, \widehat{e}_{i j q}^{*}\right\}
$$

Several combinations of error estimates (18) and (19) were tested in Ref. [15] without noticing substantial effects on the adapted resolution.

\subsection{Adaptivity algorithm}

The angular adaptivity algorithm is an iterative process described in Algorithm 1, that uses either the goal-based error measure of Eq. (20) or a regular error measure which will be defined further on in Sec. 4.1. Starting from a uniform first order discretisation $\left(W_{1,1}\right)$, the forward and adjoint problems are solved and the goal-based error measure $e_{i, j, q}$ is computed for each degree of freedom in the phase space. Theis error measure $e_{i, j, q}$ is computed for each degree of freedom in the phase space and has been scaled such that it should be less than 1.0 if the targeted tolerance on the functional has been met. If 
it is greater than 1.0, the angular resolution is locally increased by adding the basis functions of the next level of resolution that neighbour the basis function $q$ under consideration. However, if the error measure is much smaller than 1.0, here smaller than an arbitrary threshold 0.01, the angular resolution is coarsened by removing the basis function $q$.

Starting from a uniform first order angular discretisation $\left(W_{1,1}\right)$, tThe process is repeated until the adaptive angular discretisation has converged. In practice, we restrict the algorithm up to a maximum order $M$ for the Haar wavelet expansion so that the adapted resolution does not significantly evolve after $M$ iteration steps. In order to speed up the intermediate steps a reduced tolerance is applied for the convergence of the linear solver. Numerical tests have shown that this does not affect the final adapted resolution.

The angular adaptivity algorithm is applied on the coarse angular discretisation set $\overline{\mathcal{M}}$. The surjective mapping $\mathcal{C}$ from continuous nodes to discontinuous nodes (see Sec. 2.3) is used to obtain the the-sub-grid angular

discretisation set $\tilde{\mathcal{M}}$. The same set of angular basis function is used to discretise both adjoint and forward problems.

\section{Results}

In order to assess the accuracy and efficiency of the goal-based algorithm, we consider a coupled flow/radiation problem relevant for air pollution modelling in street canyons. The geometrical configuration and the boundary conditions of the problem are displayed in the left part of Fig. 3. The canyon has a reference length $L$ and contains a humid air with a homogeneous water vapour composition. Its walls are rigid, black and isothermal at $T_{\text {ref }}$ for 
$\overline{\text { Algorithm } 1 \text { AGoal-based angular adaptivity algorithm using } M \text { adaptive }}$ steps. The error measure $e_{i, j, q}$ can be either a goal-based error measure (see Sec. 3.2) or a regular error measure (see Sec. 4.1).

Set initial angular discretisation

for $m=1, \cdots, M$ do

Solve forward (Eq. (1)) and adjoint (Eq. (14)) problems

Calculate goal-based error measure (Eq. (20))

Solve forward problem (Eq. (1))

if goal-based adaptivity then

Solve adjoint problem (Eq. (14))

Calculate goal-based error measure (Eq. (20))

else if regular adaptivity then

Calculate regular error measure (Eq. (21))

end if

for $i=1, \cdots, N_{k}$ do

for $j=1, \cdots, \eta_{N}$ do

for $q \in \overline{\mathcal{M}}_{i j}$ do

if $e_{i, j, q} \mathrm{~GB}<0.01$ then

Remove angular basis function $q$ from node $j$

else if $e_{i, j, q} \stackrel{\mathrm{GB}}{\longrightarrow}>1.0$ then

Add next level angular basis function to node $j$

end if

end for

end for

end for

end for 


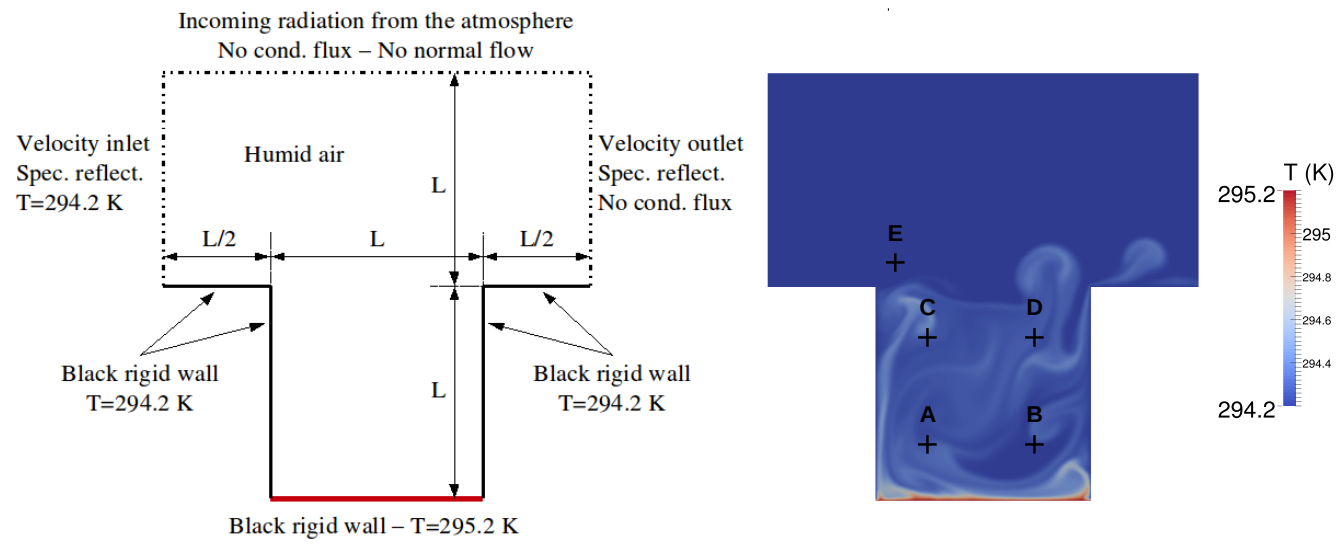

Figure 3: Schematic diagram of the street canyon configuration (left) and snapshot of the temperature field (right).

the upper and lateral walls and $T_{\text {ref }}+\Delta T$ for the ground. An isothermal inlet flow at $T_{\text {ref }}$ and $u_{\text {ref }}$ is imposed on the left open boundary while a zero conductive flux and and an outlet flow at $u_{\text {ref }}$ is imposed at the right open boundary. These two lateral open boundaries are assumed to be specular reflectors. On the top boundary, a zero normal flow and a zero conductive flux are imposed, as well as an incoming radiation flux corresponding to the flux emitted and transmitted by the atmosphere in the infrared.

This coupled flow-radiation problem is solved for Rayleigh and Reynolds numbers of $\operatorname{Ra}=g \beta \Delta T L^{3} /(\nu a)=10^{8}$ and $\operatorname{Re}=u_{\text {ref }} L / \nu=5 \times 10^{3}$, a street canyon length $L=1 \mathrm{~m}$, a reference temperature $T_{\text {ref }}=294.2 \mathrm{~K}$, a temperature difference $\Delta T=1 \mathrm{~K}$ and a water vapour content in the air of $2 \%$. The finite element fluid dynamics code Fluidity [32] is used to solve the mass, momentum and energy balance under Boussinesq approximation. A firstorder continuous $\mathbb{P}_{1}^{\mathrm{CG}}$ discretisation is applied for pressure, temperature and velocity fields and a Crank-Nicolson time integration scheme is implemented. 
The radiation field is solved with a $W_{4,4}$ uniform expansion and with $12 k$ classes to model the non-grey absorption of the humid air (see Appendix A). For both flow and radiation, the spatial domain is discretised with the samean unstructured mesh made of 22,000 continuous nodes and 130,500 discontinuous nodes, and a spatial domain decomposition was performed (48 partitions) in order to solve in parallel. An explicit coupling is carried out between flow and radiation solvers. In practice, the radiative source term is updated every 10 convection time steps. Starting from an isothermal flow at $T_{\text {ref }}$ and at rest, the coupled simulation is run for a period of $\Delta t=200 \mathrm{~s}$ in order to remove the transient features and and to reach an asymptotic unsteady regime. Tthe resulting instantaneous temperature field (snapshot) is shown in the right part of Fig. 3.

The goal-based angular adaptive algorithm is first applied in Sec. 4.1 to a single radiative transfer calculation using the coupled temperature snapshot of Fig. 3. The accuracy and the computational efficiency of the method is assessed compared to uniform angular discretisations. The distribution in space and optical thickness classes of the adapted angles is then analysed in Sec. 4.2 in order to understand which angular basis functions are removed by the method. Finally, the adapted resolution is used in the coupled simulation in Sec. 4.3 and the accuracy of the results isare again compared with uniform angular resolution.

\subsection{Accuracy and efficiency of the goal-based adaptivity method}

In order to assess the accuracy and efficiency of the goal-based adaptivity algorithm, a radiative transfer calculation from the temperature snapshot of Fig. 3 is performed with a uniform angular resolution for different Haar 
expansion order and with a goal-based adaptivity algorithm for different Haar expansion maximum order and a tolerance $\Delta \widehat{F}=0.01$. Additionally, the same calculation is done with a regular adaptivity algorithm for which the error measure does not rely on a specific target but on the value of the radiative intensity itself. Comparing goal-based and regular adapt will allow us to evaluate the relevance of the assigned goal. To perform a regular adapt calculation, we use the same algorithm 1 as for a goal-based adapt calculation but without solving the adjoint problem and replacing the goal-based error measure of Eq. (20) by the following

$$
e_{i j q}^{\mathrm{REG}}=\left|\widehat{\epsilon}_{i j q}\right| /\left(\Delta I_{i}^{\max }\right),
$$

where $\widehat{\epsilon}_{i j q}$ is the continuous angular error estimator (see Sec. 3.2) and $\Delta$ is a user-defined tolerance relative to the maximum intensity $I_{i}^{\max }$ of the $k$-class $i$ and is set to $\Delta=10^{-5}$. This tolerance value has been selected such that the regular adaptive algorithm retains around the same number of angular basis functions as the goal-based, allowing for simple comparisons.

Angular adaptivity algorithms were run in parallel but no load balancing was applied for simplicity. The spatial domain partition was performed on the first step of the adapt process, which has uniform angular resolution. However, as the number of angular basis functions varies in space when adapting, the size of the space/angle linear system assigned to each processor will differ. In order to correct this imbalance, the domain partition should be performed such that the total number of degrees of freedom (space and angle) is (roughly) the same in each domain. As the spatial distribution of the adapted angle also varies with the absorption coefficient, the domain partition would need to be updated for each $k$-class. Given we do not perform load 
balancing, the solve for each $k$-class will suffer from large load imbalances, but examining different load balancing strategies is beyond the scope of this paper and will be examined in future work.

Figure 4 shows the angular convergence of both uniform and adapted angular resolutions. The functional error is computed with respect to a reference uniform calculation $W_{4,5}$. For the uniform case, each point of the figure corresponds to a different Haar expansion order: $W_{1,1}, W_{2,2}, W_{3,3}$ and $W_{4,4}$. For the adapted cases, each point of the figure corresponds to a given maximum Haar order and number of adaptive steps: for instance, the $n^{\text {th }}$ point is obtained by running the adaptive algorithm for $n$ iterative steps up to a maximum order of $n$ in both polar and azimuthal directions and this point will be further referred to as $W_{n, n}^{\mathrm{GB}}$ or $W_{n, n}^{\mathrm{REG}}$, depending on whether goal-based or regular adaptivity is used. Comparing uniform and goal-based adapt calculations, we can observe that the goal-based adaptive algorithm keeps almost all the angular basis functions up to the second order and then discriminates the higher order angular basis functions without compromising the accuracy: the $W_{4,4}^{\mathrm{GB}}$ resolution uses five times less angular basis functions than the $W_{4,4}$ resolution to reach the same accuracy in the functional (around $0.2 \%$ ). If we now consider the regular adaptive calculation, Fig. 4 shows that the regular adaptive algorithm retains around the same number of angular basis functions than the goal-based one but with higher functional errors: the $W_{4,4}^{\mathrm{REG}}$ resolution is two times less accurate than the $W_{4,4}^{\mathrm{GB}}$ resolution for around the same number of angular basis functions $(\backsim 50)$. It confirms that the goal-based error measure derived in this paper is more appropriate than the regular one to optimise the angular resolution according to the radiative 


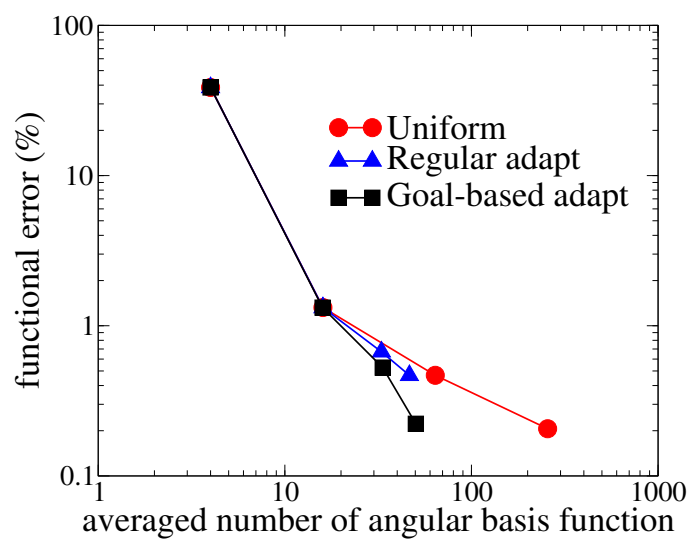

Figure 4: Functional error plot against averaged number of angular basis functions. $W_{4,5}$ reference value $F=8.8951 \times 10^{-2} \mathrm{~W} / \mathrm{m}^{3}$.

source term.

The functional errors for the same calculations are plotted in Fig. 5 against the total CPU time (wall-clock time multiplied by the number of processors). For adapted calculations, the CPU time is a cumulative time, including the computational cost of the intermediate adaptive steps. Comparing uniform and goal-based adapt calculations, we can see that the goal-based adaptive algorithm is quicker at the order 4: the $W_{4,4}^{\mathrm{GB}}$ resolution requires 5 times less CPU time than the $W_{4,4}$ resolution to reach the same accuracy in the functional, and even 6.5 times less CPU time if intermediate adaptive steps are not taken into account (in an adaptive calculation around $25 \%$ of the CPU time is spent in the intermediate steps). Not including the time of the intermediate adaptive steps allows us to assess the actual computational cost of one radiation calculation with the adapted resolution which will be used in Sec. 4.3. Comparing regular and goal-based adapt calculations, we can observe that the regular adaptive algorithm is faster and this is beause 


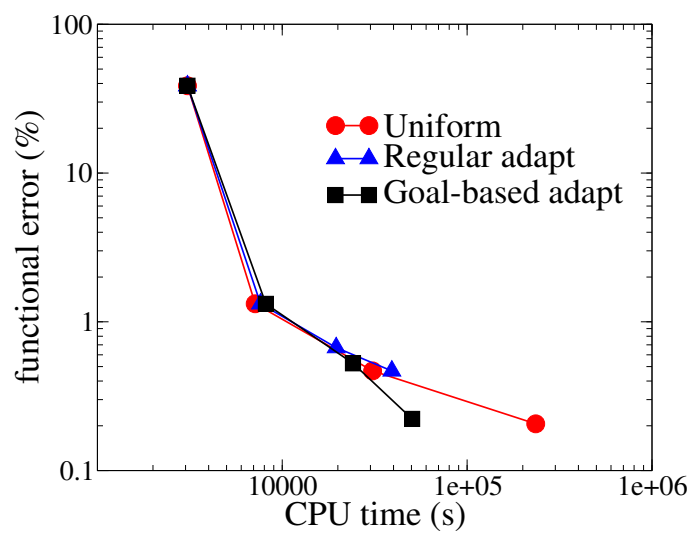

Figure 5: Functional error plot against CPU time. $W_{4,5}$ reference value $F=8.8951 \times 10^{-2} \mathrm{~W} / \mathrm{m}^{3}$.

regular adapt does not require the solve of the adjoint problem. However, the regular adapt calculation provides less accurate results: the curve follows the uniform one suggesting that there is no gain in accuracy for around the same computational cost.

So far we have assessed the accuracy in terms of the functional which is the radiative source term integrated over space. Nevertheless, we expect the computation of the radiative source term to be accurate locally in space for coupling purposes. The spatial distribution of the radiative source term is displayed in Fig. 6 together with local differences between $W_{4,4}$ and $W_{4,4}^{\mathrm{GB}}$ calculations. The radiative source term patterns are correlated to the temperature structures of Fig. 3 because of the radiation emission of the thermal hot plumes (visible in blue in the figure). A strong absorption zone (in red in the figure) is also noticeable near the bottom hot wall. The normalised differences between uniform and adapted resolutions are less than $2 \%$, confirming that the goal-based algorithm produces locally accurate results. 


\section{radiative source term $\left(\mathrm{W} / \mathrm{m}^{3}\right)$ uniform $\mathbf{W} 4,4$}

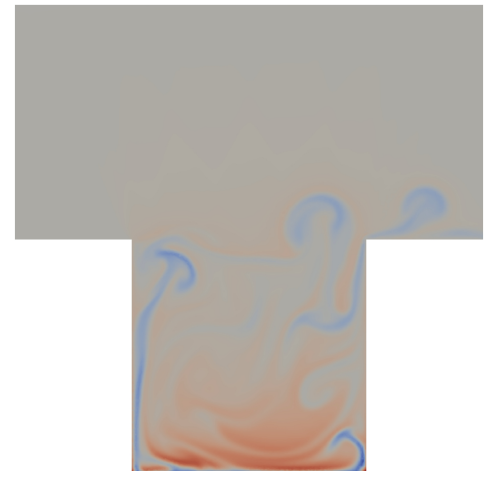

normalised difference uniform/adapt

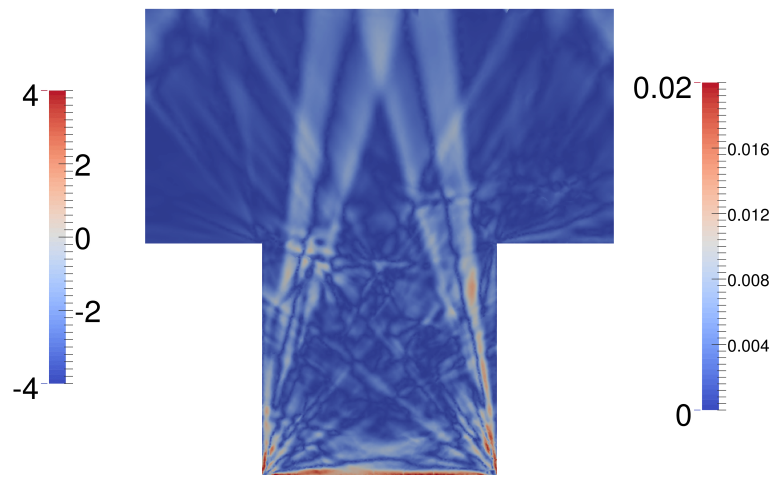

Figure 6: Radiative source term obtained with uniform angular resolution $W_{4,4}$ (left) and difference with goal-based adapted resolution $W_{4,4}^{\mathrm{GB}}$ normalised by the maximum value (right).

\subsection{Spectral and spatial distribution of the adapted angles}

The purpose of this section is to analyse which angular basis functions are retained by the goal-based adaptivity algorithm $W_{4,4}^{\mathrm{GB}}$ depending on the spatial position and the $k$-class. Figure 7 shows the number of angular basis functions, averaged in space, for each $k$-class. The distribution has a bell shape: the number of angular basis functions increases with the absorption coefficient up to a maximum reached for $k \simeq 1 \mathrm{~m}^{-1}$ and then decreases. The shape of this distribution is caused by two factors. First, in the radiative source term, the radiative intensity is weighted by the absorption coefficient which means that the higher the absorption coefficient is, the higher the contribution of the class to the source term is. This feature is captured by the source of the adjoint problem of Eq. (15) which is equal to the absorption coefficient. Secondly, the angular dependence of the radiative intensity vary 


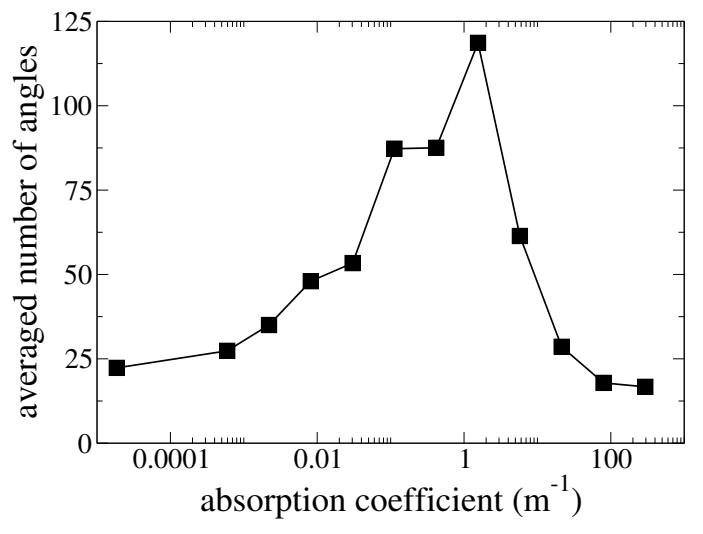

Figure 7: Averaged number of angular basis functions for each $k$-class of the global model for goal-based adapt $W_{4,4}^{\mathrm{GB}}$.

with optical thickness, and we know that in thick media, the radiative transfer becomes local and the radiative intensity is close to isotropic. We can thus infer that a low number of angular basis functions is sufficient to accurately represent the radiative intensity for the thick classes.

This explanation is confirmed by the spatial distribution of the number of angular basis functions, shown in Fig. 8 for three different classes. For the thickest class $\left(k_{10}=21 \mathrm{~m}^{-1}\right)$, a small number of angular basis functions is used throughout the domain except near the non-reflecting boundaries, where the radiative intensity is more likely to deviate from isotropic. Although the number of angular basis functions is also quite low in average for the thinnest class $\left(k_{3}=2.2 \times 10^{-3} \mathrm{~m}^{-1}\right)$, its spatial distribution strongly differs. Marks of ray effect can be observed that suggests that the radiative intensity is highly directional within the domain and probably under-resolved. Finally, the number of angular basis functions is the highest for the intermediate class $\left(k_{6}=0.11 \mathrm{~m}^{-1}\right)$, and its spatial distribution is rather homogeneous except 

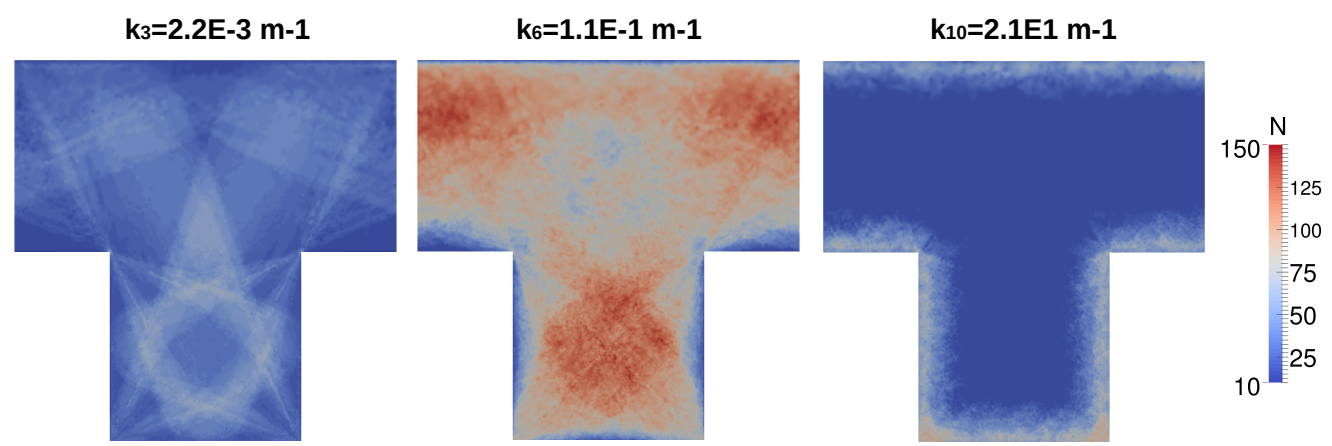

Figure 8: Spatial distribution of the number of angular basis functions for three different $k$-class for goal-based adapt $W_{4,4}^{\mathrm{GB}}$.

near the boundaries.

In order to further the analysis, the angular distribution of the radiative intensity at spatial points A and E (see locations in Fig. 3) and for two classes $\left(k_{4}=8.2 \times 10^{-3} \mathrm{~m}^{-1}\right.$ and $\left.k_{8}=1.55 \mathrm{~m}^{-1}\right)$ is depicted in Fig. 9. Both uniform $W_{4,4}$ and adapted $W_{4,4}^{\mathrm{GB}}$ are shown, allowing us to assess how well the adapted angular resolution captures the angular flux. Adapted results satisfactorily reproduce the uniform ones but larger differences are noticed for the thinner class $\left(k_{4}\right)$, which do not need to be resolved accurately to compute the source term. The angular distribution of the intensity is dominated by the boundary conditions. The angular shape depends on how the lateral walls, the bottom wall and the atmosphere are seen from the given points A (within the canyon) or $\mathrm{E}$ (outside the canyon). The angular shape also differs between the two $k$-classes in two ways. First, the flux coming from the atmosphere is lower than the flux coming from the lateral walls for the thinner class $\left(k_{4}\right)$ while the two are equal for the thicker class $\left(k_{8}\right)$. Secondly, there is no polar variation for the thinner class while the flux decreases with the polar angle because of 

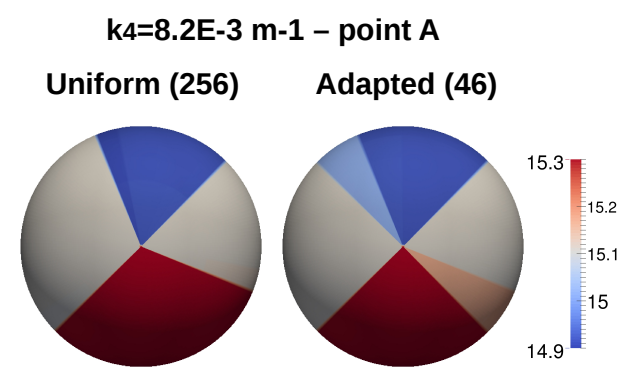

$k 8=1.55 \mathrm{~m}-1$ - point A
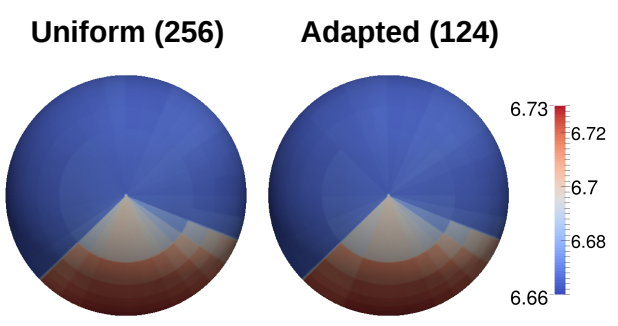

$\mathrm{k} 4=8.2 \mathrm{E}-3 \mathrm{~m}-1$ - point $\mathrm{E}$
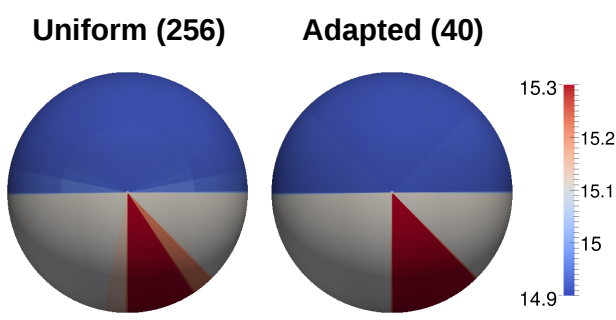

$\mathrm{k} 8=1.55 \mathrm{~m}-1$ - point $\mathrm{E}$

Uniform (256)

Adapted (88)
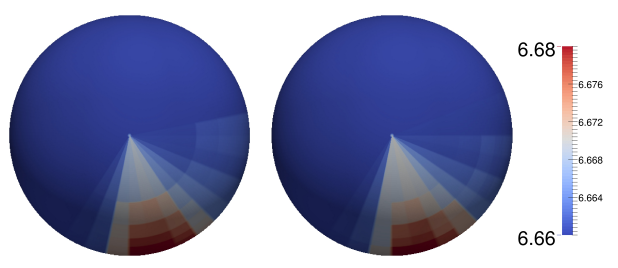

Figure 9: Angular distribution of the radiative intensity at different spatial points and $k$ class. Comparison between uniform $W_{4,4}$ and goal-based adapt $W_{4,4}^{\mathrm{GB}}$ results. The location of points $\mathrm{A}$ and $\mathrm{E}$ are given in Fig. 3. The number in parenthesis indicates the number of angular basis functions.

radiation extinction for the thicker class.

\subsection{Coupled calculations}

In the previous section we demonstrated that the goal-based algorithm is useful in efficiently computing the radiative source term from a temperature snapshot. The analysis is now focused on the practical use of the adapted angular resolution in flow-radiation coupled calculations. For this purpose, the coupled calculations are carried on with two different angular discretisation for radiation: one with uniform Haar wavelet $W_{4,4}$ and one with the adapted resolution $W_{4,4}^{\mathrm{GB}}$. This comparison is shown in Fig. 10, which plots the normalised differences on the radiative source term over time between 
the two calculations at different spatial points inside the canyon (see locations in Fig. 3). The agreement between the two is good over the whole integration period, although the differences seem to increase with time. This is an expected result as the differences in the radiative source term impact the temperature calculation and thus the differences accumulate.

Numerical tests were performed to check if it might be beneficial to change the adapted resolution over time by running the goal-based algorithm after a few radiation calculations. The question was if the angular resolution was affected by the local temperature field and thus needed to follow its time evolution. Compared to keeping the initial adapted resolution, no significant gain in accuracy was obtained. Indeed, the spatial distribution of the adapted angles (Fig. 8) does not seem to be correlated with the temperature field (Fig. 3) and was thus not substantially varying over time. Moreover, the angular distributions displayed in Fig. 9 tend to show that the angular dependence of the radiation field is dominated by the boundary conditions, rather than determined by the local physical properties. This would probably not be true if the temperature of the boundaries were changing over time.

\section{Conclusion}

In this paper, we have presented a goal-based angular adaptivity method suitable for radiation modelling in non grey media when the radiation field is coupled with an unsteady flow. The method optimises the angular resolution according to the radiative source term (the goal) which is the key physical quantity for the coupling between flow and thermal radiation. The angular 


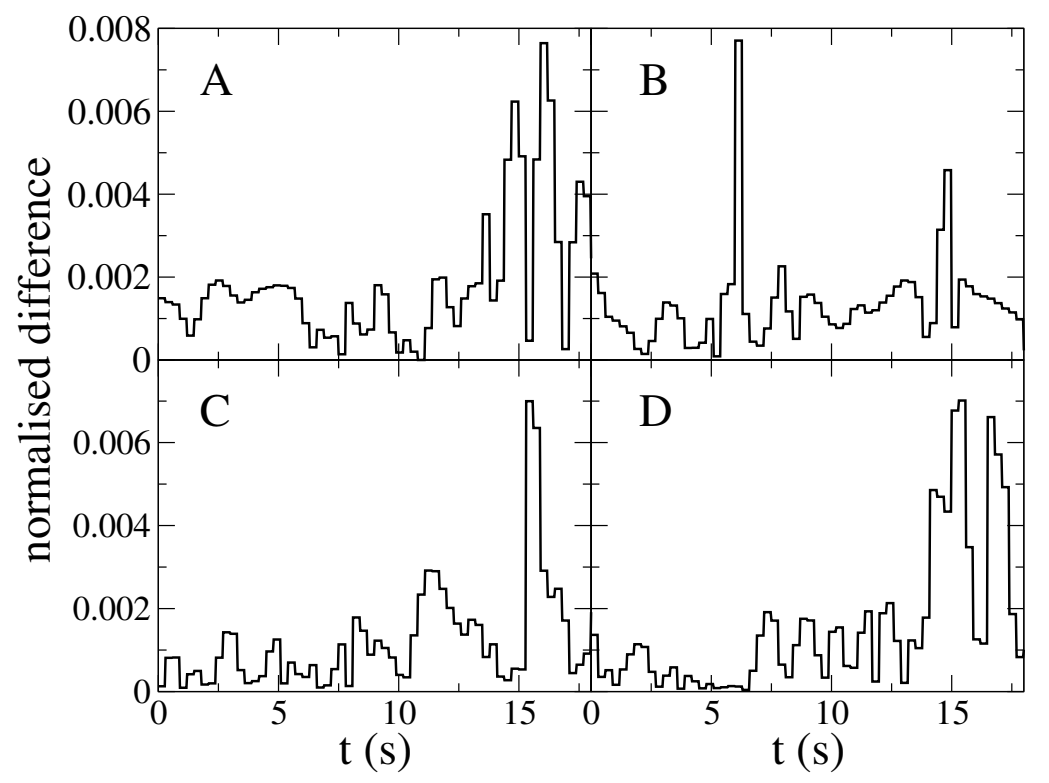

Figure 10: Difference in the radiative source term between uniform $W_{4,4}$ and adapted $W_{4,4}^{\mathrm{GB}}$ resolution during a coupled unsteady simulation at different spatial points. Differences are normalised according to the maximum value of the source term across space and time. The location of points A, B, C and D is given in Fig. 3. 
resolution is allowed to vary anisotropically in space and for each absorption coefficient class built from a global model of the radiative properties of the medium. The angular discretisation is based on a Haar wavelet expansion, which is a hierarchical version of the discrete ordinates expansion and does not require any angular interpolation in space when adapting.

The method has been tested on a coupled flow-radiation problem relevant for air pollution modelling in street canyons. Compared to a uniform Haar wavelet resolution, a goal-based adapted resolution requires 5 times less angular basis functions and 6.5 less CPU time to reach the same level of accuracy in the radiative source term. In addition, the adapted resolution proved to be accurate when used in unsteady coupled calculations. The gain in computational time will help us to investigate larger computational domains and more realistic flow regimes that involves a larger range of temporal and spatial scales. In future work, we plan to further enhance the computational efficiency of the adapted calculations by performing load balancing in parallel after adapting, to ensure an even distribution of work.

Another perspective to this work would be to extend the application of the method to heterogeneous media that are encountered for instance in combustion processes. It would be then necessary to take into account the spatial variations of the absorption coefficient with the temperature and the composition of the radiating gases (water vapour and carbon dioxide). However, the goal-based algorithm would not be affected because it will still be possible to develop a global model of the radiative properties of such media and to assign to each $k$-class a different angular resolution. 
Acknowledgments This work used the ARCHER UK National Supercomputing Service (http://www.archer.ac.uk). Dr Laurent Soucasse acknowledges support from the European Commission's Framework Programme Horizon 2020, through the Marie Skodowska-Curie Individual Fellowship Grant Agreement 659442. Dr Andrew Buchan acknowledges funding through the EPSRC grant ref.: EP/M022864/1.

\section{Appendix A. Global model for radiative properties}

The Line-By-Line (LBL) spectrum of the absorption coefficient of the mixture considered in Sec. 4 (humid air with $2 \%$ water vapour) has been computed with the HITRAN 2008 database [33] with a resolution of $0.025 \mathrm{~cm}^{-1}$ and is shown in the left part of Fig. A.11. Following Eq. (4), the corresponding absorption distribution function has been calculated at $T_{\text {ref }}=294.2 \mathrm{~K}$ and the values of the absorption coefficient were logarithmically discretised between $k_{\min }=1.36 \times 10^{-6} \mathrm{~m}^{-1}$ and $k_{\max }=5.83 \times 10^{2} \mathrm{~m}^{-1}$ into 12 consecutive ranges. The accuracy of the model compared to the LBL approach is examined from emissivity $\varepsilon_{\text {tot }}$ calculation of homogeneous columns of different lengths $l$ at $T_{\text {ref }}$ given by

$$
\varepsilon_{\text {tot }}=\frac{\pi}{\sigma T_{\text {ref }}^{4}} \int_{0}^{\infty} I_{b}\left(\nu, T_{\text {ref }}\right)\left(1-\exp \left(\kappa_{\nu} l\right)\right) d \nu \simeq \sum_{i=1}^{N_{k}} a_{i}\left(1-\exp \left(k_{i} l\right)\right) .
$$

Results are shown in the right part of Fig. A.11 where it can be seen a very good agreement.

The incoming flux boundary condition of the street canyon problem in Sec. 4 has been computed at high spectral resolution using the atmospheric radiation code LBLRTM-LW [34] for a mid-latitude summer atmosphere, 

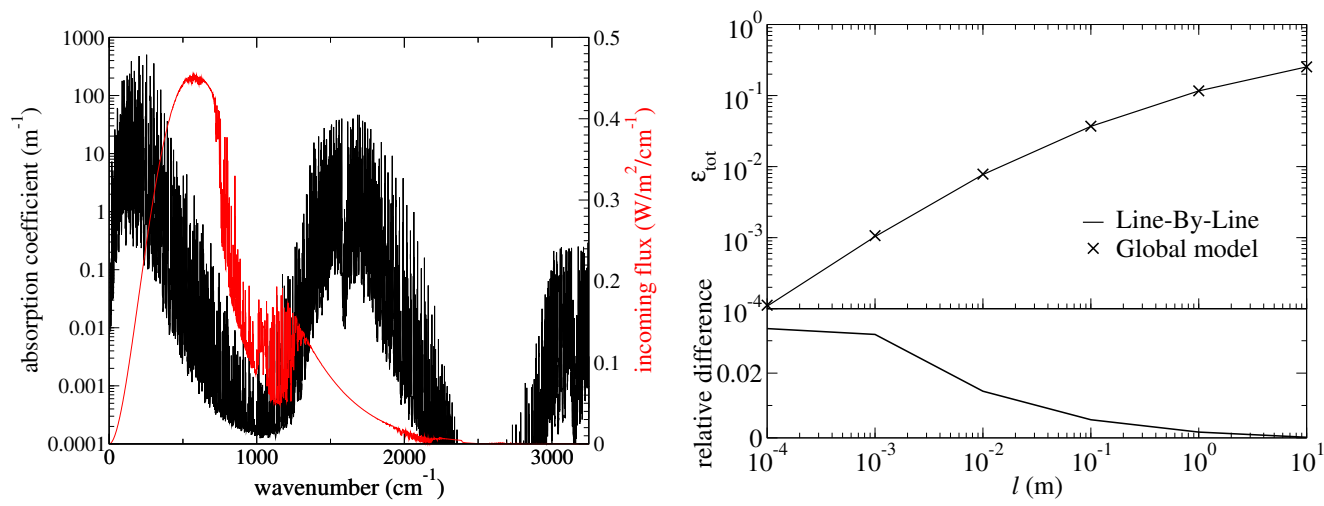

Figure A.11: Left: LBL absorption coefficient of the humid air mixture at atmospheric pressure $\left(X_{\mathrm{ref}}^{\mathrm{H}_{2} \mathrm{O}}=0.02\right.$ and $\left.T_{\text {ref }}=294.2 \mathrm{~K}\right)$ and LBL incoming flux for a mid-latitude summer atmosphere. Right: Spectrally integrated emissivities for different homogeneous column lengths calculated with LBL and global models at $X_{\text {ref }}^{\mathrm{H}_{2} \mathrm{O}}$ and $T_{\text {ref }}$.

with a resolution of $1 \mathrm{~cm}^{-1}$ and is plotted in the left part of Fig. A.11. The spectral distribution of the flux follows a blackbody distribution at $T_{\text {ref }}=294.2 \mathrm{~K}$ with holes corresponding to the transparency zones of the atmosphere. In order to correlate this spectral distribution with the global radiative property model, the LBL incoming flux $I^{\text {inc }}(\nu)$ needs to be reordered as a function of the values of the absorption coefficient $I^{\text {inc }}(k)$ following

$$
I^{\text {inc }}(k)=\frac{\partial}{\partial k} \int_{\nu, \kappa_{\nu}\left(T_{\text {ref }}\right) \leq k} I^{\text {inc }}(\nu) d \nu .
$$

The incoming flux $I_{i}^{\text {inc }}$ for each $k$-class $i$ of our discretisation is then given by

$$
I_{i}^{\mathrm{inc}}=\int_{\nu, \kappa_{\nu}\left(T_{\mathrm{ref}}\right) \leq k_{i}^{+}} I^{\mathrm{inc}}(\nu) d \nu-\int_{\nu, \kappa_{\nu}\left(T_{\mathrm{ref}}\right) \leq k_{i}^{-}} I^{\mathrm{inc}}(\nu) d \nu,
$$

where $k_{i}^{+}$and $k_{i}^{-}$are the upper and lower bounds of the absorption coefficient values of the $k$-class $i$. 


\section{References}

[1] L. Soucasse, Ph. Rivière, A. Soufiani, S. Xin, P. Le Quéré, Transitional regimes of natural convection in a differentially heated cavity under the effects of wall and molecular gas radiation, Physics of Fluids 26 (2014) 024105.

[2] J. Taine, A. Soufiani, Gas IR radiative properties: from spectroscopic data to approximate models, Advances in Heat Transfer 33 (1999) 295414.

[3] R. Capdevila, C. D. Pérez-Segarra, A. Oliva, Development and comparison of different spatial numerical schemes for the radiative transfer equation resolution using three-dimensional unstructured meshes, Journal of Quantitative Spectroscopy and Radiative Transfer 111 (2010) 264-273.

[4] P. J. Coelho, Advances in the discrete ordinates and finite volume methods for the solution of radiative heat transfer problems in participating media, review, Journal of Quantitative Spectroscopy and Radiative Transfer 145 (2014) 121-146.

[5] R. O. Castro, J. P. Trelles, Spatial and angular finite element method for radiative transfer in participating media, Journal of Quantitative Spectroscopy and Radiative Transfer 157 (2015) 81105.

[6] F. Ogando, P. Velarde, Development of a radiation transport fluid dynamic code under AMR scheme, Journal of Quantitative Spectroscopy and Radiative Transfer 71 (2001) 541-550. 
[7] J. C. Ragusa, A simple Hessian-based 3D mesh adaptation technique with applications to the multigroup diffusion equations, Annals of $\mathrm{Nu}-$ clear Energy 35 (2008) 20062018.

[8] D. Lathouwers, Goal-oriented spatial adaptivity for the $S_{N}$ equations on unstructured triangular meshes, Annals of Nuclear Energy 38 (2011) 1373-1381.

[9] M. A. Goffin, C. M. J. Baker, A. G. Buchan, C. C. Pain, M. D. Eaton, P. N. Smith, Minimising the error in eigenvalue calculations involving the Boltzmann transport equation using goal-based adaptivity on unstructured meshes, Journal of Computational Physics 242 (2013) 726-752.

[10] R. Yang, G. Yuan, $h$-Refinement for simple corner balance scheme of $S_{N}$ transport equation on distorted meshes, Journal of Quantitative Spectroscopy and Radiative Transfer 184 (2016) 241-253.

[11] R. T. Ackroyd, W. E. Wilson, Composite finite element solutions for neutron transport, Annals of Nuclear Energy 15 (8) (1988) 397-419.

[12] M. A. Goffin, A. G. Buchan, A. C. Belme, C. C. Pain, M. D. Eaton, P. N. Smith, R. P. Smedley-Stevenson, Goal-based angular adaptivity applied to the spherical harmonics discretisation of the neutral particle transport equation, Annals of Nuclear Energy 71 (2014) 6080.

[13] A. G. Buchan, C. C. Pain, M. D. Eaton, R. P. Smedley-Stevenson, A. J. H. Goddard, Linear and quadratic octahedral wavelets on the sphere for angular discretisations of the Boltzmann transport equation, Annals of Nuclear Energy 32 (2005) 12241273. 
[14] A. M. Watson, The $W_{N}$ adaptive method for numerical solution of particle transport problems, Ph.D. thesis, Texas A\&M University (2005).

[15] M. A. Goffin, A. G. Buchan, S. Dargaville, C. C. Pain, P. N. Smith, R. P. Smedley-Stevenson, Goal-based angular adaptivity applied to a wavelet-based discretisation of the neutral particle transport equation, Journal of Computational Physics 281 (2015) 1032-1062.

[16] J. C. Stone, Adaptive discrete-ordinates algorithms and strategies, Ph.D. thesis, Texas A\&M University (2007).

[17] J. Kópházi, D. Lathouwers, A space-angle DGFEM approach for the Boltzmann radiation transport equation with local angular refinement, Journal of Computational Physics 297 (2015) 637-668.

[18] A. G. Buchan, A. A. Calloo, M. G. Goffin, S. Dargaville, F. Fang, C. C. Pain, I. M. Navon, A POD reduced order model for resolving angular direction in neutron/photon transport problems, Journal of Computational Physics 296 (2015) 138157.

[19] J. Tencer, K. Carlberg, R. Hogan, M. Larsen, Reduced order modelling applied to the discrete ordinates method for radiation heat transfer in participating media, HT2016-7010, in: Proceedings of the 2016 Summer Heat Transfer Conference, ASME, Washington, DC, USA, 2016.

[20] M. K. Denison, B. W. Webb, An absorption-line blackbody distribution function for efficient calculation of total gas radiative transfer, Journal of Quantitative Spectroscopy and Radiative Transfer 50 (5) (1993) 499510. 
[21] M. K. Denison, B. W. Webb, The spectral-line-based weighted-sum-ofgray-gases model in nonisothermal nonhomogeneous media, Journal of Heat Transfer 117 (1995) 359-365.

[22] L. Pierrot, Ph. Rivière, A. Soufiani, J. Taine, A fictitious-gas-based absorption distribution function global model for radiative transfer in hot gases, Journal of Quantitative Spectroscopy and Radiative Transfer 62 (1999) 609-624.

[23] M. F. Modest, H. Zhang, The full-spectrum correlated-k distribution for thermal radiation from molecular gas-particulate mixtures, Journal of Heat Transfer 124 (2002) 30-38.

[24] T. J. R. Hughes, G. Scovazzi, P. Bochev, A. Buffa, A multiscale discontinuous Galerkin method with the computational structure of a continuous Galerkin method, Computer Methods in Applied Mechanics and Engineering 195 (2006) 2761-2787.

[25] A. G. Buchan, A. S. Candy, S. R. Merton, C. C. Pain, J. I. Haidi, M. D. Eaton, A. J. H. Goddard, R. P. Smedley-Stevenson, The innerelement subgrid scale finite element method for the Boltzmann transport equation, Nuclear Science and Engineering 164 (2010) 105-121.

[26] A. G. Buchan, C. C. Pain, An efficient space-angle subgrid scale discretisation of the neutron transport equation, Annals of Nuclear Energy 94 (2016) 440-450.

[27] B. J. Adigun, A. G. Buchan, A. Adam, S. Dargaville, M. A. Goffin, C. C. 
Pain, A Haar wavelet method for angularly discretising the Boltzmann transport equation, submitted to Progress in Nuclear Energy.

[28] A. G. Buchan, S. R. Merton, C. C. Pain, R. P. Smedley-Stevenson, Riemann boundary conditions for the Boltzmann transport equation using arbitrary angular approximations, Annals of Nuclear Energy 38 (2011) 1186-1195.

[29] A. Adam, A. G. Buchan, M. D. Piggott, C. C. Pain, J. Hill, M. A. Goffin, Adaptive Haar wavelets for the angular discretisation of spectral wave models, Journal of Computational Physics 305 (2016) 521538.

[30] A. G. Buchan, C. C. Pain, A. P. Umpleby, R. P. Smedley-Stevenson, A sub-grid scale finite element agglomeration multigrid method with application to the Boltzmann transport equation, International Journal for Numerical Methods in Engineering 92 (2012) 318-342.

[31] L. Soucasse, Ph. Rivière, S. Xin, P. Le Quéré, A. Soufiani, Numerical study of coupled molecular gas radiation and natural convection in a differentially heated cubical cavity, Computational Thermal Sciences 4 (2012) 335-350.

[32] C. C. Pain, A. P. Umpleby, C. R. E. de Oliveira, A. J. H. Goddard, Tetrahedral mesh optimisation and adaptivity for steady-state and transient finite element calculations, Computer Methods in Applied Mechanics and Engineering 190 (2001) 37713796.

[33] L. S. Rothman, I. E. Gordon, A. Barbe, D. Chris Benner, P. F. Bernath, M. Birk, V. Boudon, L. R. Brown, A. Campargue, J. P. Champion, 
K. Chance, L. H. Coudert, V. Dana, V. M. Devi, S. Fally, J. M. Flaud, R. R. Gamache, A. Goldman, D. Jacquemart, I. Kleiner, N. Lacome, W. J. Lafferty, J. Y. Mandin, S. T. Massie, S. N. Mikhailenko, C. E. Miller, N. Moazzen-Ahmadi, O. V. Naumenko, A. V. Nikitin, J. Orphal, V. I. Perevalov, A. Perrin, A. Predoi-Cross, C. Rinsland, M. Rotger, M. Šimečková, M. A. H. Smith, K. Sung, S. A. Tashkun, J. Tennyson, R. A. Toth, A. C. Vandaele, J. Vander Auwera, The HITRAN 2008 molecular spectroscopic database, Journal of Quantitative Spectroscopy and Radiative Transfer 110 (2009) 533-572.

[34] S. A. Clough, M. W. Shephard, E. J. Mlawer, J. S. Delamere, M. J. Iacono, K. Cady-Pereira, S. Boukabara, P. D. Brown, Atmospheric radiative transfer modeling: a summary of the AER codes, short communication, Journal of Quantitative Spectroscopy and Radiative Transfer 91 (2005) 233-244. 